\title{
Report on Quadrupole and Dipole Sorting for the APS Booster Synchrotron
}

\author{
Rabinder Kumar Koul \\ APS, Argonne National Laboratory \\ Argonne, IL 60439
}

10V 145

OSTI

\begin{abstract}
This is a report of the implementation of dipole and quadrupole magnet sorting carried out for the injector synchrotron. The method used for implementing the sorting was developed by the author, see Koul ${ }^{1}$ or $\mathrm{Koul}^{2}$. The arrangement of the dipoles around the injector synchrotron, after sorting, reduced the $\delta x / \sqrt{\beta}$ by a factor of six. Whereas the arrangement of the quadrupoles around the injector synchrotron, after sorting, reduced $\delta \beta / \beta$ by approximately a factor of five.
\end{abstract}

\section{Introduction}

The APS injector synchrotron ring measures approximately 368 meters in circumference. The ring is designed to accelerate the positrons from the initial energy of $450 \mathrm{MeV}$ to the final energy of about $7.5 \mathrm{GeV}$, at low beam loss. It has sixty-eight dipole magnets and eighty quadrupole magnets. The field tolerance limits on these magnets were given earlier from linearized error analysis, and reported in $\mathrm{Koul}^{3}$ or Teng ${ }^{4}$. These limits were further verified by simulations carried out using the computer program Elegant, see Koul ${ }^{5}$. The tolerance

This manuscript has been authored under contract number W31-109-ENG-38 with the U.S. Department of Energy. Accordingly, the U.S. Government retains a non-exclusive, royalty-free license to publish or reproduce the published form of this contribution, or allow others to do so, for U.S. Government purposes. 


\section{DISCLAIMER}

This report was prepared as an account of work sponsored by an agency of the United States Government. Neither the United States Government nor any agency thereof, nor any of their employees, make any warranty, express or implied, or assumes any legal liability or responsibility for the accuracy, completeness, or usefulness of any information, apparatus, product, or process disclosed, or represents that its use would not infringe privately owned rights. Reference herein to any specific commercial product, process, or service by trade name, trademark, manufacturer, or otherwise does not necessarily constitute or imply its endorsement, recommendation, or favoring by the United States Government or any agency thereof. The views and opinions of authors expressed herein do not necessarily state or reflect those of the United States Government or any agency thereof. 


\section{DISCLAIMER}

Portions of this document may be illegible electronic image products. Images are produced from the best available original document. 
limit for dipoles was established at $\triangle B / B \sim 10^{-3}$, whereas for quadrupoles the quadrupole error tolerance was given at $\Delta B^{\prime} / B^{\prime} \sim 1.5 \times 10^{-3}$.

In the following we will first report on the sorting of the dipoles and then on the sorting of the quadrupoles.

\section{Dipole Sorting:}

The measured magnetic field data for the injector synchrotron dipoles was provided by the magnet measurement group. The data used in the sorting procedure is shown in the fourth coloumn of Table 1. The sorting of the dipoles was carried out in four phases. In the first phase sixteen magnets were sorted. Random errors for the magnets were obtained by subtracting the mean of the error, obtained for the first twenty-one magnets measured, from the measured errors. The mean value of errors for the subsequent batches was obtained by using all the magnets measured, i.e., both previously sorted and the newly measured magnets. Figure 1 shows how this mean kept changing. To sort the subsequent batches, the new value of the random errors was used for the previously sorted magnets. This problem of changing mean error values rendered the sorting process less than ideal. However, the results of this sorting show that this technique is efficient even in such situations. The orbit distortion $\delta x / \sqrt{\beta}$ was reduced by a factor of six as compared to the orbit distortion one would have obtained if the magnets were not sorted. The list of the sorted magnets is given in Table 1. Position one corresponds to the dipole magnet $\mathrm{B} 1 \mathrm{C} 1 \mathrm{~B} 1$, with succeeding dipoles following in the clockwise direction. The first column of the table gives the position assigned to a dipole, named in column two. The third column gives the corresponding position of the dipole magnet in the injector synchrotron. Finally the fourth column gives the measured error value used for final sorting.

\section{Quadrupole Sorting}

The quadrupoles were also sorted in batches. However, the mean value of the error from the first batch to the last batch changed by an order of magnitude, see figure 2. Such a large change in the mean value made the previous sorted batch very ineffective in reducing the amplification factor of the succeeding sort. 
In view of this, it was decided that the final sort must be carried out on the remaining 40 quadrupoles simultaneously. This helped and $\delta \beta / \beta$ was reduced by a factor of 4.6 over a random placement. The final placement of the sorted quadrupoles is given in Table 2. As in the case of first table, the first column gives the position assigned to the quadrupole named in column two, the third column gives the corresponding position in the injector synchrotron, and the last column gives the measured error value of the quadrupole used in sorting.

\section{References:}

1. Rabinder Kumar Koul, AIP Conference Proceedings No 292; Particles and Fields Series 54; Stability of Particle Motion in Storage Rings, 1992.

2. R. K. Koul, Fredrick Lopez and Fredrick E. Mills, Optimal Magnet Sorting Procedure and Application to the APS Injector Synchrotron, Proc. of the 1993 IEEE Particle Accelerator Conference, held in Washington, DC, May 1993.

3. R. K. Koul and F. E. Mills, Linearized Error Analysis for an Accelerator and Application to the APS Injector Synchrotron, LS-230, Dec. 1993.

4. L. Teng, private communication.

5. R. K. Koul, to be published. 
Table 1: SORTED BOOSTER DIPOLES:

\begin{tabular}{|l|c|c|c|}
\hline Assigned Position & $\begin{array}{c}\text { Dipole } \\
\text { Name }\end{array}$ & $\begin{array}{c}\text { Booster Position } \\
\text { Name }\end{array}$ & $\begin{array}{c}\text { Random Error } \\
\text { Value }\end{array}$ \\
\hline POSITION[1] & BDP017 & B1C1B1 & 0.0000839130434782607 \\
POSITION[2] & BDP009 & B1C2B1 & 0.000003913043478260647 \\
POSITION[3] & BDP010 & B1C2B2 & 0.0003139130434782607 \\
POSITION[4] & BDP014 & B1C3B1 & 0.0001539130434782607 \\
POSITION[5] & BDP033 & B1C3B2 & 0.0003039130434782606 \\
POSITION[6] & BDP035 & B1C4B1 & 0.0003939130434782606 \\
POSITION[7] & BDP031 & B1C4B2 & 0.0005839130434782608 \\
POSITION[8] & BDP008 & B1C5B1 & -0.00002608695652173933 \\
POSITION[9] & BDP013 & B1C5B2 & -0.0003760869565217392 \\
POSITION[10] & BDP004 & B1C6B1 & 0.0003839130434782607 \\
POSITION[11] & BDP012 & B!C6B2 & 0.0001839130434782607 \\
POSITION[12] & BDP021 & B1C7B1 & -0.0001560869565217393 \\
POSITION[13] & BDP029 & B1C7B2 & 0.00007391304347826067 \\
POSITION[14] & BDP019 & B1C8B1 & 0.0003539130434782606 \\
POSITION[15] & BDP022 & B1C8B2 & 0.00003391304347826072 \\
POSITION[16] & BDP026 & B1C9B1 & 0.0002039130434782606 \\
POSITION[17] & BDP015 & B1C9B2 & 0.0003839130434782607 \\
POSITION[18] & BDP024 & B2C0B1 & 0.0000439130434782607 \\
POSITION[19] & BDP027 & B2C0B2 & 0.0001739130434782607 \\
POSITION[20] & BDP028 & B2C1B1 & 0.0003839130434782607 \\
POSITION[21] & BDP018 & B2C1B2 & -0.0001960869565217393 \\
POSITION[22] & BDP030 & B2C2B1 & 0.0003639130434782607 \\
POSITION[23] & BDP034 & B2C2B2 & 0.0000439130434782607 \\
\hline
\end{tabular}




\begin{tabular}{|c|c|c|c|}
\hline Assigned Position & $\begin{array}{l}\text { Dipole } \\
\text { Name }\end{array}$ & $\begin{array}{c}\text { Booster Position } \\
\text { Name }\end{array}$ & $\begin{array}{c}\text { Random Error } \\
\text { Value }\end{array}$ \\
\hline POSITION[24] & BDP051 & $\mathrm{B} 2 \mathrm{C} 3 \mathrm{~B} 1$ & 0.0004239130434782607 \\
\hline POSITION[25] & BDP020 & $\mathrm{B} 2 \mathrm{C} 3 \mathrm{~B} 2$ & 0.0002039130434782606 \\
\hline POSITION[26] & $\mathrm{BDP} 023$ & $\mathrm{~B} 2 \mathrm{C} 4 \mathrm{~B} 1$ & 0.00005391304347826065 \\
\hline POSITION[27] & BDP032 & $\mathrm{B} 2 \mathrm{C} 4 \mathrm{~B} 2$ & 0.00007391304347826067 \\
\hline POSITION[28] & BDP036 & $\mathrm{B} 2 \mathrm{C} 5 \mathrm{~B} 1$ & 0.0001739130434782607 \\
\hline POSITION[29] & $\mathrm{BDP} 025$ & $\mathrm{~B} 2 \mathrm{C} 5 \mathrm{~B} 2$ & 0.0001039130434782606 \\
\hline POSITION[30] & BDP011 & $\mathrm{B} 2 \mathrm{C} 6 \mathrm{~B} 1$ & 0.0001639130434782607 \\
\hline POSITION[31] & BDP016 & $\mathrm{B} 2 \mathrm{C} 6 \mathrm{~B} 2$ & 0.0001839130434782607 \\
\hline POSITION[32] & BDP059 & $\mathrm{B} 2 \mathrm{C} 7 \mathrm{~B} 1$ & -0.0007260869565217393 \\
\hline POSITION[33] & BDP045 & $\mathrm{B} 2 \mathrm{C} 7 \mathrm{~B} 2$ & 0.0004039130434782608 \\
\hline POSITION[34] & BDP054 & $\mathrm{B} 2 \mathrm{C} 8 \mathrm{~B} 2$ & 0.0002139130434782607 \\
\hline POSITION[35] & BDP062 & $\mathrm{B} 3 \mathrm{C} 1 \mathrm{~B} 1$ & -0.0005560869565217395 \\
\hline POSITION[36] & BDP043 & $\mathrm{B} 3 \mathrm{C} 2 \mathrm{~B} 1$ & 0.000003913043478260647 \\
\hline POSITION[37] & BDP047 & $\mathrm{B} 3 \mathrm{C} 2 \mathrm{~B} 2$ & 0.00007391304347826067 \\
\hline POSITION[38] & BDP070 & $\mathrm{B} 3 \mathrm{C} 3 \mathrm{~B} 1$ & -0.0004560869565217395 \\
\hline POSITION[39] & BDP007 & $\mathrm{B} 3 \mathrm{C} 3 \mathrm{~B} 2$ & -0.000936086956521739 \\
\hline POSITION[40] & BDP040 & $\mathrm{B} 3 \mathrm{C} 4 \mathrm{~B} 1$ & 0.0002039130434782607 \\
\hline POSITION[41] & BDP060 & $\mathrm{B} 3 \mathrm{C} 4 \mathrm{~B} 2$ & -0.0005360869565217394 \\
\hline POSITION[42] & BDP053 & $\mathrm{B} 3 \mathrm{C} 5 \mathrm{~B} 1$ & 0.0001139130434782607 \\
\hline POSITION[43] & BDP071 & $\mathrm{B} 3 \mathrm{C} 5 \mathrm{~B} 2$ & -0.0007560869565217394 \\
\hline POSITION[44] & BDP041 & $\mathrm{B} 3 \mathrm{C} 6 \mathrm{~B} 1$ & 0.0003539130434782607 \\
\hline POSITION[45] & BDP068 & $\mathrm{B} 3 \mathrm{C} 6 \mathrm{~B} 2$ & -0.0005560869565217395 \\
\hline POSITION[46] & BDP050 & $\mathrm{B} 3 \mathrm{C} 7 \mathrm{B1}$ & 0.0002239130434782607 \\
\hline
\end{tabular}




\begin{tabular}{|c|c|c|c|}
\hline Assigned Position & $\begin{array}{l}\text { Dipole } \\
\text { Name }\end{array}$ & $\begin{array}{l}\text { Booster Position } \\
\text { Name }\end{array}$ & $\begin{array}{c}\text { Random Error } \\
\text { Value }\end{array}$ \\
\hline POSITION[47] & BDP042 & $\mathrm{B} 3 \mathrm{C} 7 \mathrm{~B} 2$ & 0.0001239130434782607 \\
\hline POSITION[48] & BDP039 & B3C8B1 & 0.0000139130434782607 \\
\hline POSITION[49] & BDP058 & $\mathrm{B} 3 \mathrm{C} 8 \mathrm{~B} 2$ & -0.0006760869565217394 \\
\hline POSITION $[50]$ & BDP055 & $\mathrm{B} 3 \mathrm{C} 9 \mathrm{~B} 1$ & -0.0003260869565217392 \\
\hline POSITION[51] & BDP064 & $\mathrm{B} 3 \mathrm{C} 9 \mathrm{~B} 2$ & -0.0003360869565217394 \\
\hline POSITION[52] & BDP049 & $\mathrm{B} 4 \mathrm{C} 0 \mathrm{~B} 1$ & $0: 0002639130434782607$ \\
\hline POSITIÒN[53] & BDP005 & $\mathrm{B} 4 \mathrm{COB} 2$ & 0.0005639130434782608 \\
\hline POSITION[54] & BDP038 & $\mathrm{B} 4 \mathrm{C} 1 \mathrm{~B} 1$ & -0.00007608695652173934 \\
\hline POSITION[55] & $\mathrm{BDP} 057$ & $\mathrm{~B} 4 \mathrm{C} 1 \mathrm{~B} 2$ & -0.0001660869565217393 \\
\hline POSITION[56] & BDP056 & $\mathrm{B} 4 \mathrm{C} 2 \mathrm{~B} 1$ & -0.0001060869565217393 \\
\hline POSITION[57] & $\mathrm{BDP} 044$ & $\mathrm{~B} 4 \mathrm{C} 2 \mathrm{~B} 2$ & 0.0003139130434782607 \\
\hline POSITION[58] & $\mathrm{BDP} 067$ & $\mathrm{~B} 4 \mathrm{C} 3 \mathrm{~B} 1$ & -0.0001060869565217393 \\
\hline POSITION[59] & BDP066 & $\mathrm{B} 4 \mathrm{C} 3 \mathrm{~B} 2$ & -0.0003660869565217393 \\
\hline POSITION[60] & $\mathrm{BDP} 048$ & $\mathrm{~B} 4 \mathrm{C} 4 \mathrm{~B} 1$ & 0.0001939130434782607 \\
\hline POSITION[61] & $\mathrm{BDP} 052$ & $\mathrm{~B} 4 \mathrm{C} 4 \mathrm{~B} 2$ & -0.0001660869565217393 \\
\hline POSITION[62] & BDP006 & $\mathrm{B} 4 \mathrm{C} 5 \mathrm{~B} 1$ & -0.000826086956521739 \\
\hline POSITION[63] & BDP037 & $\mathrm{B} 4 \mathrm{C} 5 \mathrm{~B} 2$ & 0.0001339130434782607 \\
\hline POSITION[64] & BDP065 & $\mathrm{B} 4 \mathrm{C} 6 \mathrm{~B} 1$ & -0.00005608695652173932 \\
\hline POSITION[65] & BDP061 & $\mathrm{B} 4 \mathrm{C} 6 \mathrm{~B} 2$ & -0.0004360869565217394 \\
\hline POSITION[66] & BDP069 & $\mathrm{B} 4 \mathrm{C} 7 \mathrm{~B} 1$ & -0.0005560869565217395 \\
\hline POSITION[67] & BDP046 & $\mathrm{B} 4 \mathrm{C} 7 \mathrm{~B} 2$ & 0.0002239130434782607 \\
\hline POSITION[68] & BDP063 & $\mathrm{B} 4 \mathrm{C} 8 \mathrm{~B} 2$ & -0.0003360869565217394 \\
\hline
\end{tabular}




\section{MEAN VALUE AS FUNCTION OF BATCH NUMBER}

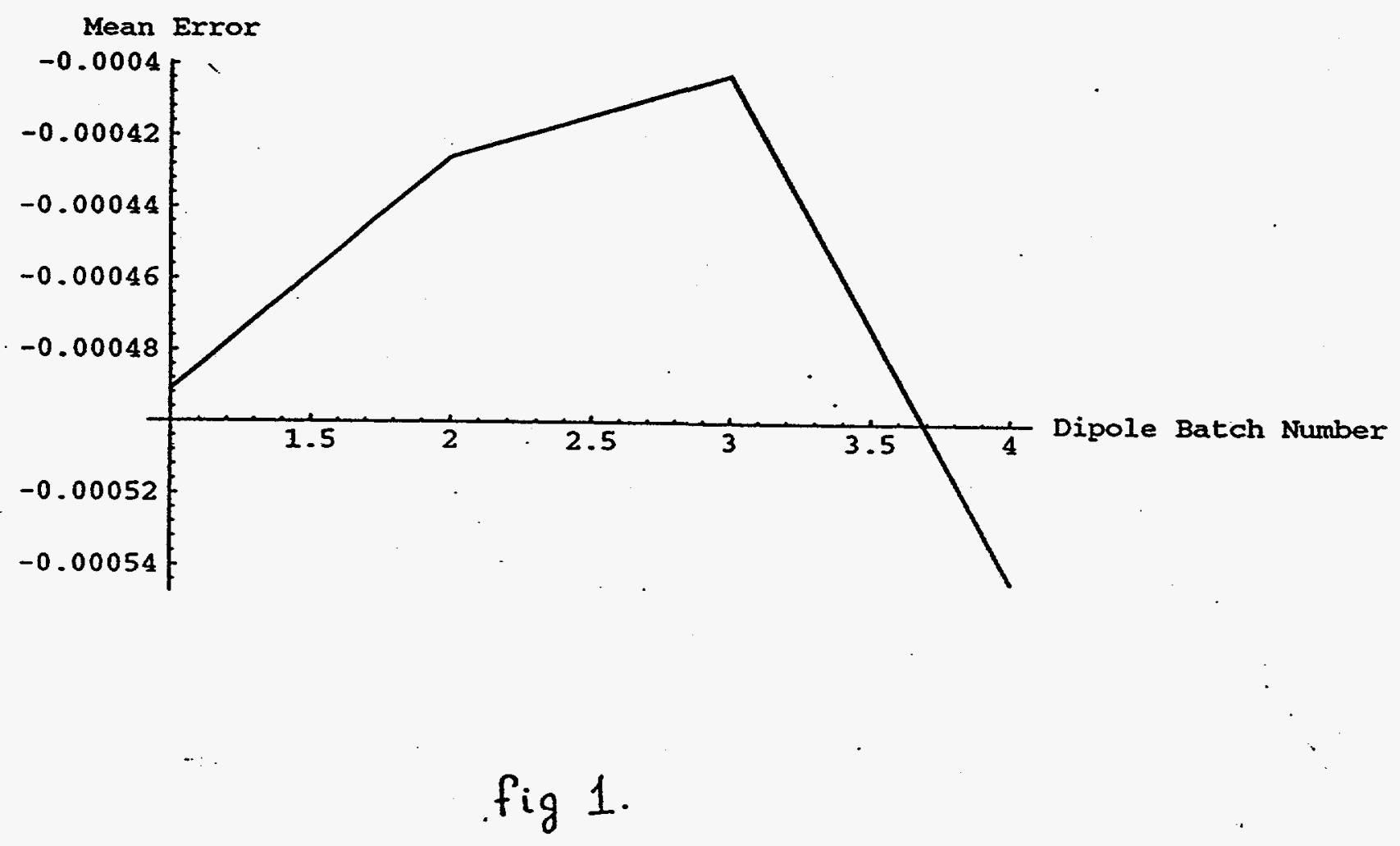


MEAN VALUE AS a fUNCTION OF BATCH NUMBER

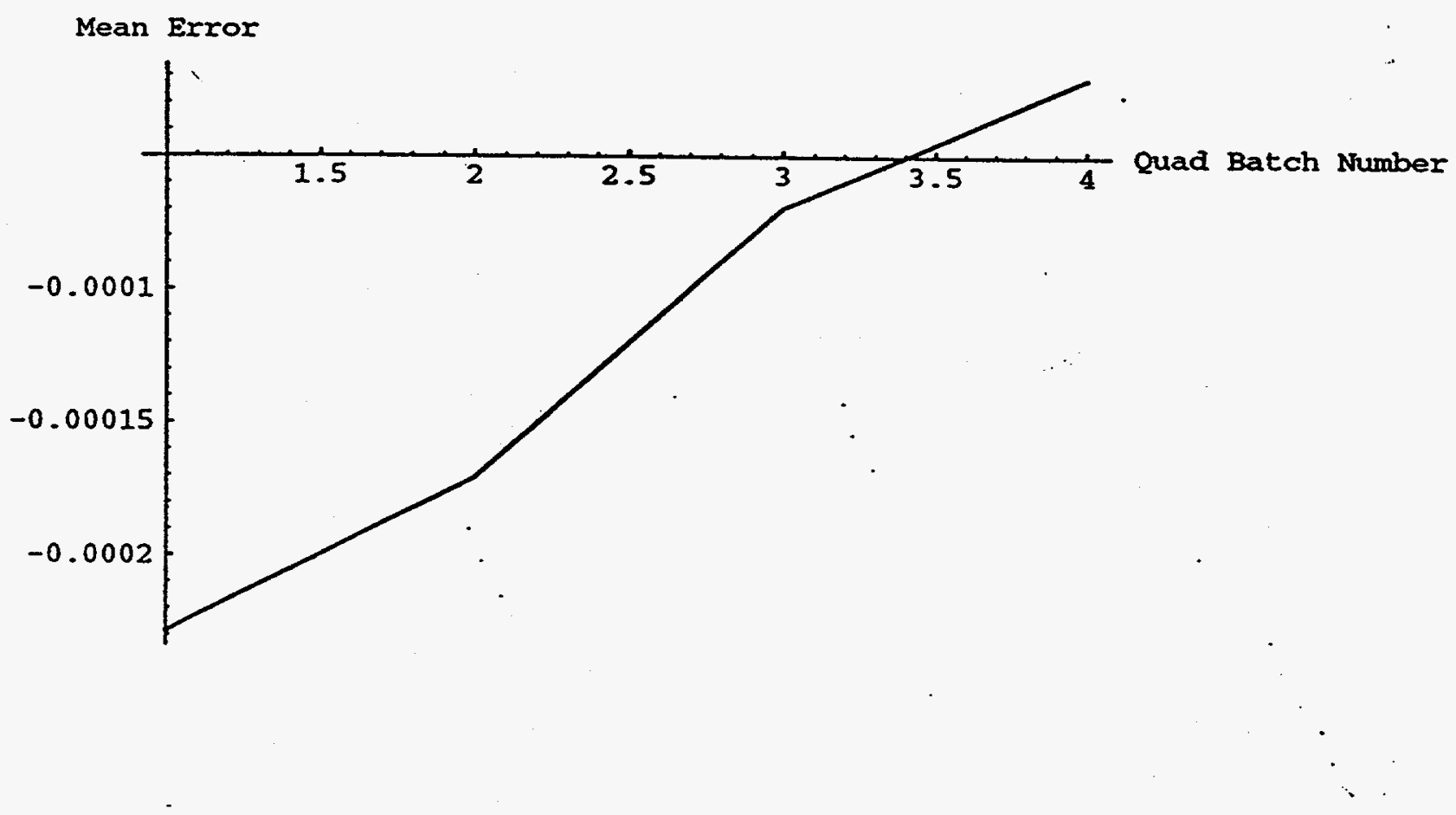

fig 2. 
Table 2: SORTED BOOSTER QUARDRUPOLES:

\begin{tabular}{|l|c|c|c|}
\hline Assigned Position & $\begin{array}{c}\text { Quadrupole } \\
\text { Name }\end{array}$ & $\begin{array}{c}\text { Booster Position } \\
\text { Name }\end{array}$ & $\begin{array}{c}\text { Random Error } \\
\text { Value }\end{array}$ \\
\hline POSITION[1] & BQP022 & B2C8QF & -0.0001060330120481927 \\
POSITION[2] & BQP033 & C7QD & -0.0003497130120481928 \\
POSITION[3] & BQP021 & QF & -0.0002422030120481927 \\
POSITION[4] & BQP027 & C6QD & -0.0002063730120481928 \\
POSITION[5] & BQP019 & QF & -0.0002135430120481927 \\
POSITION[6] & BQP004 & C5QD & -0.0002923730120481927 \\
POSITION[7] & BQP009 & QF & 0.00003730698795180725 \\
POSITION[8] & BQP020 & C4QD & -0.0003783830120481928 \\
POSITION[9] & BQP014 & QF & -0.0001347030120481927 \\
POSITION[10] & BQP017 & C3QD & -0.0005002130120481928 \\
POSITION[11] & BQP013 & QF & -0.0002637130120481927 \\
POSITION[12] & BQP008 & C2QD & 0.0001233069879518073 \\
POSITION[13] & BQP012 & QF & -0.0002278730120481928 \\
POSITION[14] & BQP011 & C1QD & -0.0005647230120481927 \\
POSITION[15] & BQP010 & QF & 0.00003730698795180725 \\
POSITION[16] & BQP006 & C0QD & 0.0004314869879518073 \\
POSITION[17] & BQP025 & QF & -0.0002063730120481928 \\
POSITION[18] & BQP016 & B1C9QD & 0.0005246569879518073 \\
POSITION[19] & BQP005 & QF & 0.000001466987951807253 \\
POSITION[20] & BQP023 & C8QD & -0.0006292230120481928 \\
\hline
\end{tabular}




\begin{tabular}{|l|c|c|c|}
\hline Assigned Position & $\begin{array}{c}\text { Quadrupole } \\
\text { Name }\end{array}$ & $\begin{array}{c}\text { Booster Position } \\
\text { Name }\end{array}$ & $\begin{array}{c}\text { Random Error } \\
\text { Value }\end{array}$ \\
\hline POSITION[21] & BQP031 & QF & 0.0002594769879518072 \\
POSITION[22] & BQP048 & C7QD & -0.0001777030120481926 \\
POSITION[23] & BQP026 & QF & -0.0007295530120481928 \\
POSITION[24] & BQP041 & C6QD & 0.0001376369879518072 \\
POSITION[25] & BQP032 & QF & -0.0007940630120481929 \\
POSITION[26] & BQP038 & C5QD & 0.0002666469879518073 \\
POSITION[27] & BQP053 & QF & 0.0004601469879518075 \\
POSITION[28] & BQP030 & C4QD & -0.0002852130120481928 \\
POSITION[29] & BQP015 & QF & 0.0004099869879518073 \\
POSITION[30] & BQP029 & C3QD & 0.00004446698795180727 \\
POSITION[31] & BQP036 & QF & -0.0005288830120481928 \\
POSITION[32] & BQP054 & C2QD & 0.0001018069879518073 \\
POSITION[33] & BQP037 & QF & -0.0005002130120481928 \\
POSITION[34] & BQP042 & C1QD & -0.0002565430120481928 \\
POSITION[35] & BQP039 & QF & -0.0006793930120481928 \\
POSITION[36] & BQP034 & C0QD & 0.0002308069879518072 \\
POSITION[37] & BQP045 & QF & 0.0003598169879518073 \\
POSITION[38] & BQP028 & B4C9QD & 0.0003454769879518072 \\
POSITION[39] & BQP035 & QF & 0.0005246569879518073 \\
POSITION[40] & BQP044 & C8QD & 0.0002594769879518072 \\
\hline
\end{tabular}




\begin{tabular}{|l|c|c|c|}
\hline Assigned Position & $\begin{array}{c}\text { Quadrupole } \\
\text { Name }\end{array}$ & $\begin{array}{c}\text { Booster Position } \\
\text { Name }\end{array}$ & $\begin{array}{c}\text { Random Error } \\
\text { Value }\end{array}$ \\
\hline POSITION[41] & BQP024 & QF & 0.001004836987951807 \\
POSITION[42] & BQP065 & C7QD & 0.0005533169879518073 \\
POSITION[43] & BQP040 & QF & -0.000980403012048193 \\
POSITION[44] & BQP051 & C6QD & 0.0003239769879518072 \\
POSITION[45] & BQP057 & QF & 0.0005748169879518074 \\
POSITION[46] & BQP069 & C5QD & 0.0003454769879518072 \\
POSITION[47] & BQP066 & QF & 0.0005819869879518074 \\
POSITION[48] & BQP073 & C4QD & 0.0005461569879518074 \\
POSITION[49] & BQP060 & QF & 0.000839996987951807 \\
POSITION[50] & BQP052 & C3QD & 0.0001878069879518072 \\
POSITION[51] & BQP043 & QF & -0.0003927130120481928 \\
POSITION[52] & BQP068 & C2QD & 0.0003239769879518072 \\
POSITION[53] & BQP050 & QF & 0.0005891569879518073 \\
POSITION[54] & BQP083 & C1QD & 0.0001304769879518072 \\
POSITION[55] & BQP077 & QF & 0.0006751569879518074 \\
POSITION[56] & BQP070 & C0QD & 0.00003730698795180725 \\
POSITION[57] & BQP055 & QF & -0.0004930530120481929 \\
POSITION[58] & BQP063. & B3C9QD & 0.0002738069879518072 \\
POSITION[59] & BQP082 & QF & -0.0004428830120481928 \\
POSITION[60] & BQP067 & C8QD & 0.00005880698795180727 \\
\hline
\end{tabular}




\begin{tabular}{|c|c|c|c|}
\hline Assigned Position & $\begin{array}{c}\text { Quadrupole } \\
\text { Name }\end{array}$ & $\begin{array}{c}\text { Booster Position } \\
\text { Name }\end{array}$ & $\begin{array}{c}\text { Random Error } \\
\text { Value }\end{array}$ \\
\hline POSITION[61] & BQP018 & QF & 0.0007396569879518072 \\
\hline POSITION[62] & BQP080 & C7QD & -0.00004870301204819274 \\
\hline POSITION[63] & BQP047 & $Q F$ & 0.0007683269879518074 \\
\hline POSITION[64] & BQP078 & C6QD & 0.0004171469879518073 \\
\hline POSITION[65] & BQP058 & $Q F$ & $-0.000184873012048192 \dot{7}$ \\
\hline POSITION[66] & BQP059 & C5QD & 0.0000874669879518073 \\
\hline POSITION[67] & BQP061 & QF & -0.000837063012048193 \\
\hline POSITION[68] & BQP071 & C4QD & 0.0006608269879518075 \\
\hline POSITION[69] & BQP075 & $\mathrm{QF}$ & 0.0007038269879518074 \\
\hline POSITION[70] & BQP007 & C3QD & 0.00007313698795180727 \\
\hline POSITION[71] & BQP079 & QF & -0.0001562030120481928 \\
\hline POSITION[72] & BQP049 & $\mathrm{C} 2 \mathrm{QD}$ & 0.0001233069879518073 \\
\hline POSITION[73] & BQP074 & $\mathrm{QF}$ & 0.0005461569879518074 \\
\hline POSITION[74] & BQP056 & C1QD & 0.0001663069879518072 \\
\hline POSITION[75] & BQP076 & QF & -0.0001203730120481927 \\
\hline POSITION[76] & BQP072 & $\mathrm{COQD}$ & 0.00005163698795180726 \\
\hline POSITION[77] & BQP081 & $Q F$ & -0.0001418730120481928 \\
\hline POSITION[78] & BQP003 & $\mathrm{B} 2 \mathrm{C} 9 \mathrm{QD}$ & 0.0005031569879518073 \\
\hline POSITION[79] & $\mathrm{BQP} 062$ & QF & -0.0006220530120481928 \\
\hline POSITION[80] & BQP064 & $\mathrm{C} 8 \mathrm{QD}$ & -0.000917030120481928 \\
\hline
\end{tabular}

